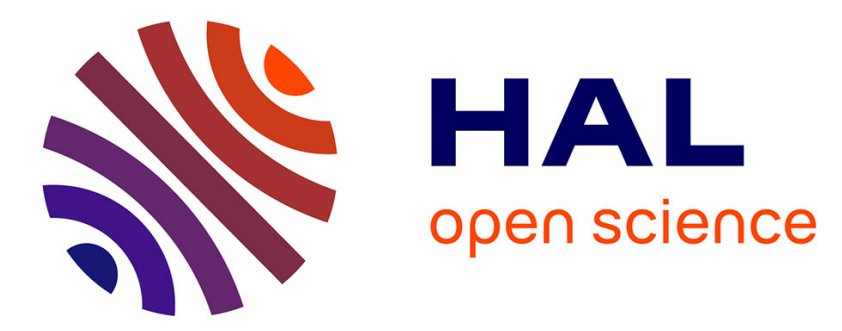

\title{
A Web-based Dashboard for the High-level Monitoring of ALMA
}

Emmanuel Pietriga, Giorgio Filippi, Luis Véliz, Fernando del Campo, Jorge

Ibsen

\section{- To cite this version:}

Emmanuel Pietriga, Giorgio Filippi, Luis Véliz, Fernando del Campo, Jorge Ibsen. A Web-based Dashboard for the High-level Monitoring of ALMA. SPIE Astronomical Telescopes and Instrumentation 2014, SPIE, Jun 2014, Montréal, Canada. pp.1B:1-1B:12, 10.1117/12.2055235 hal-01061544

\section{HAL Id: hal-01061544 \\ https://hal.inria.fr/hal-01061544}

Submitted on 7 Sep 2014

HAL is a multi-disciplinary open access archive for the deposit and dissemination of scientific research documents, whether they are published or not. The documents may come from teaching and research institutions in France or abroad, or from public or private research centers.
L'archive ouverte pluridisciplinaire $\mathbf{H A L}$, est destinée au dépôt et à la diffusion de documents scientifiques de niveau recherche, publiés ou non, émanant des établissements d'enseignement et de recherche français ou étrangers, des laboratoires publics ou privés. 


\title{
A Web-based Dashboard for the High-level Monitoring of ALMA
}

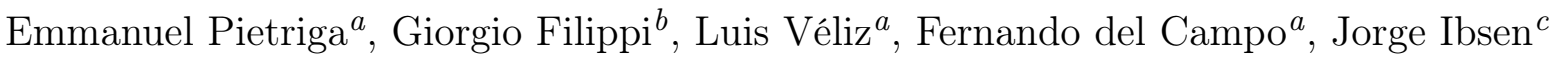 \\ ${ }^{a}$ INRIA Chile - CIRIC, Santiago, Chile; \\ ${ }^{b}$ ESO, Garching, Germany; \\ ${ }^{c}$ Joint ALMA Office, Santiago, Chile
}

\begin{abstract}
The ALMA radio-telescope's operations depend on the availability of high-level, easy-to-understand status information about all of its components. The ALMA Dashboard aims at providing an all-in-one-place near-real-time overview of the observatory's key elements and figures to both line and senior management. The Dashboard covers a wide range of elements beyond antennas, such as pads, correlator and central local oscillator. Data can be displayed in multiple ways, including: a table view, a compact view fitting on a single screen, a timeline showing detailed information over time, a logbook, a geographical map.
\end{abstract}

Keywords: ALMA, Radio-telescope, Operations Monitoring, Dashboard, Human-Computer Interaction, Data Visualization

\section{INTRODUCTION}

The ALMA radio-telescope is the largest millimeter/submillimeter radio astronomy observatory in the world, at an altitude of 5,000 meters in the high Atacama desert of Chile. With spatial and spectral resolution two orders of magnitude better than that of existing facilities, ALMA gives astronomers unprecedented views of star and planet formation in our galaxy and of the early Universe. It is a complex instrument made of many different components: antennas, correlator, pads, power generation and distribution network, etc. Its operations depend on the availability of high-level, easy-to-understand status information about all of these components. We have developed a Web-based application called the ALMA Dashboard, that both provides an overview of the observatory's status and allows users to get a more detailed report about particular components. The ALMA Dashboard does not intend to replace the specialized real-time monitoring and reporting subsystem tools used in the control room. ${ }^{1-3}$ It rather aims at providing an easily accessible, all-in-one-place near-real-time overview of the observatory's key elements and figures to both line and senior management.

The Dashboard features views on the data that can be tailored according to different user profiles, from a high-level overview of the instrument's performance to a detailed view of each antenna's primary components. Interactive queries that can be made on the data include, e.g., how many antennas are currently available for science, or more elaborate ones such as how many antennas feature fully operational B4 and B6 receivers in the array's central cluster. Query results can be displayed in multiple ways, including: a table view, a compact view fitting on a single screen, a geographical map. Addition views are available, such as a timeline showing the status of the main components of an antenna over time, and a logbook.

The Dashboard initially focused on the observatory's 66 antennas. Following the successful deployment and use of this first version, it is now getting extended to cover a wider range of elements beyond antennas and their constitutive components, such as pads holding the antennas, correlator and central local oscillator, and the power network. One of the Dashboard's main design goals is to add little-to-no overhead to the existing work processes, by aggregating data from manual user input, from existing software systems, and as a by-product of organizational processes.

Further author information: (Send correspondence to Emmanuel Pietriga)

Emmanuel Pietriga: E-mail: emmanuel.pietriga@inria.fr, Giorgio Filippi: E-mail: gfilippi@eso.org, Luis Véliz: E-mail: luis.veliz@inria.cl, Fernando del Campo: E-mail: fernando.delcampo@inria.cl, Jorge Ibsen: E-mail: jibsen@alma.cl 
Following a user-centered design process similar to the one adopted for the design of real-time visualization components for the ALMA control room, ${ }^{1}$ representatives of the different Dashboard user groups - including astronomers, operators and engineers - have been working jointly with human-computer interaction researchers on the design of the Dashboard.

In this paper, we describe the method employed to inform the Dashboard's user interface design. We then give an overview of the first version of the Dashboard, followed by a description of the extensions and improvements currently being developed. Information about the technical implementation follows, and we conclude with some words about other ALMA systems now making use of Dashboard data in other contexts, such as the control room for manual array creation.

\section{METHOD}

Antennas are the main components of the ALMA radio-observatory. Their availability and characteristics (what receivers are operational, where is the antenna located, etc.) have a direct impact on what observations can be performed. It was thus decided to focus the Dashboard primarily on those elements during the first phase of its development. The Dashboard took inspiration from the ad-hoc tools that were in use during construction to track the status of antennas delivered to the Array Operations Site (AOS), which included a collaborative spreadsheet that was used by various teams to track the primary components of each antenna. An analysis of these tools and discussions with their users helped identify the relevant pieces of data captured in those status tracking tools and the work processes associated with them. A set of requirements and a first user interface design was elaborated on this basis and iterated upon with a subgroup of representative target end-users (December 2012). This initial design is described in Section 3. Following the successful deployment of this first version (April 2013), which eventually led to the withdrawal of some of the above-mentioned ad-hoc tools and their replacement by the Dashboard, several iterations were made to make improvements. It was then decided to start a second phase of development, extending the scope of the Dashboard beyond antennas, adding alternate views on the antenna status data, and improving user interaction with the system. This second phase (initiated in October 2013 and planned for delivery in the third quarter of 2014), described in Section 4, was essentially driven by a user-centered design approach, which involved:

- a participatory design workshop organized by the HCI researchers and engineers, involving a small group of end-users (operators, astronomers, etc.) who are the domain-expert end-users of the Dashboard;

- in-situ interviews with ALMA operators, engineers and astronomers;

- rapid low-fidelity prototyping of ideas resulting from this workshop;

- discussions on this basis with stakeholders, gathering feedback and new ideas;

- high-quality implementation of the ideas identified through this process and incremental delivery and deployment of novel features over several months.

Each category of actors plays an essential role. Operators and astronomers have significant domain expertise, they are well aware of the context-of-work, the associated requirements, and the needs of end-users. They contribute ideas, and can quickly provide feedback about the relevance of different options. HCI researchers are best aware of the state-of-the-art, and can suggest possible solutions to a given problem that other members of the group who are less familiar with the latest research and development advances in the field might not be aware of. Finally, ALMA and INRIA engineers also participate in the brainstorming, suggesting solutions and later in the process providing feedback about the feasibility of the considered options. The participatory design workshop was organized in sessions that focused on well-identified topics. Each session was limited to a small number of people, taken from a total of 18 participants depending on the subject at hand. Overall, the workshop lasted 1.5 days and included a half-dozen focus sessions, so as to ensure that participants were highly committed and actively contributed to the discussions and idea generation process.

From the very beginning, and given the wide variety of end-users targeted by the Dashboard, it was decided to develop it as a Web-based application to facilitate access to it from any device (workstation, handheld device such as tablets and smartphones), without requiring users to install specific software components on their computer. 


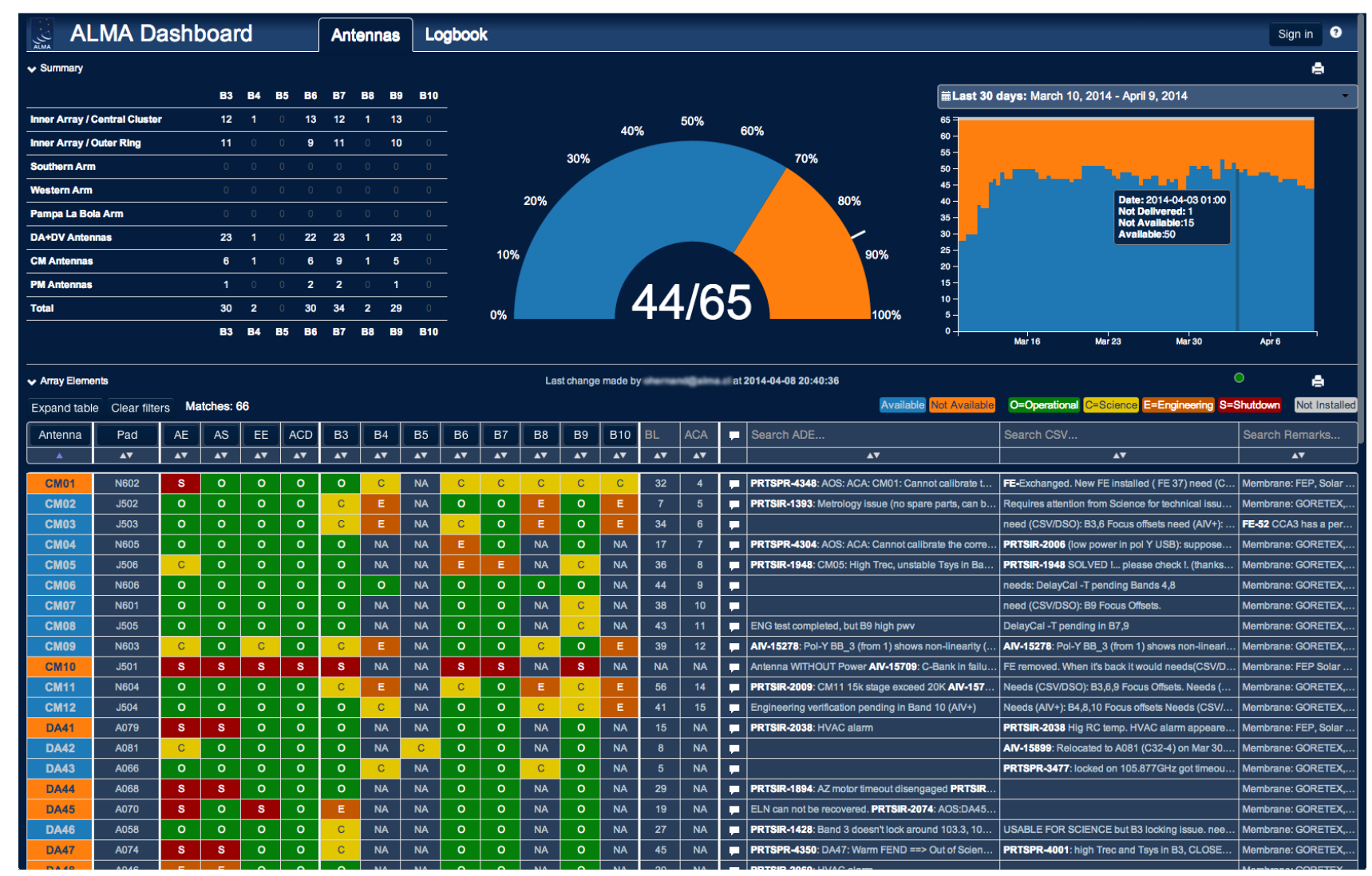

Figure 1. Dashboard main page, giving an overview of antenna availability in the upper part (Summary), and a breakdown of the primary components' operational status for each antenna (Array Elements).

\section{AN ANTENNA-CENTRIC DASHBOARD}

The Dashboard's main page (Figure 1) is organized in two main parts: the top part gives an overview of antenna availability for scientific observations at the AOS, while the lower part provides more detailed information, indicating the operational status of each antenna's primary components. The information is organized in a table, reminiscent of the original spreadsheet, each row corresponding to an antenna. The table also serves as the main entry point to access and modify the detailed status of each antenna. The Dashboard also provides mechanisms to navigate back in time and see the observatory's status at an earlier date, as detailed below.

\subsection{Overview of Antennas}

The upper part of Figure 1 shows the Overview section of the Dashboard's main page (Summary). It is designed to provide users with a high-level summary of the observatory's status, from an antenna-centric perspective.

The chart in the middle shows in a prominent manner the current number of antennas available for science. In this example, 44 out of the 65 antennas delivered to the AOS are considered as usable to perform scientific observations. An antenna's availability for science is automatically determined based on a logical expression that computes this overall status based on the operational status of that antenna's primary components.

The stacked area chart on the right shows the evolution of this figure over some time span that can be changed interactively by users, ranging from the last few hours to several years. Its purpose is both to indicate the trend at different time scales, and to act as a quick shortcut to navigate back in time, as detailed in Section 3.5. While the use of stacked area charts as opposed to line charts is debatable, we opted for the former in this particular case because the total number of antennas is constant and only three statuses exist: available (blue $\square$ ), not

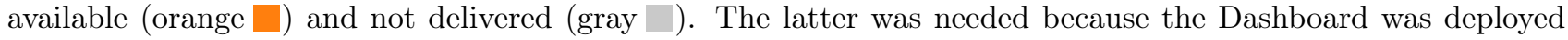
while some of the antennas had not yet been handed over for scientific operational use. In the near future, this 


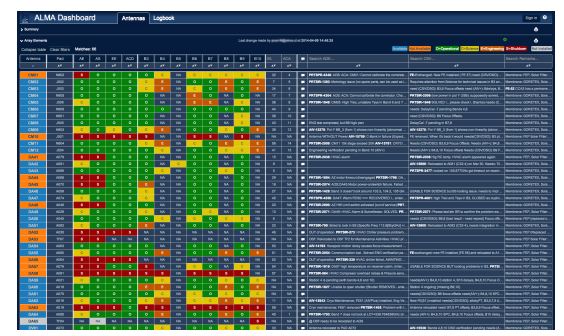

(a)

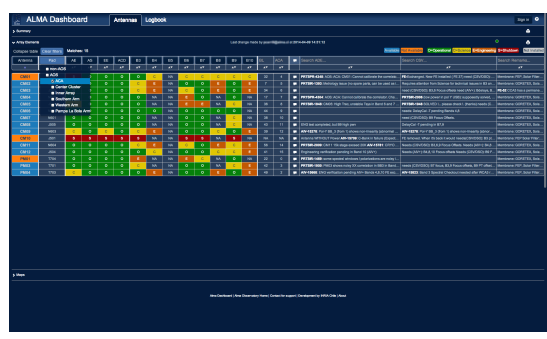

(b)

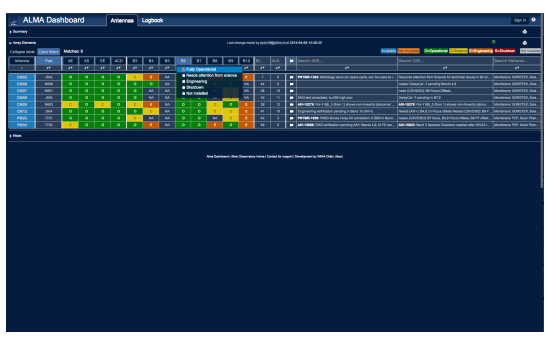

(c)

Figure 2. Filtering the antenna table: (a) full complement of antennas, (b) showing only antennas from the Atacama Compact Array, (c) further filtering this list to show only antennas from the Atacama Compact Array that have receiver for B6 fully operational.

particular status may be omitted, or used for another purpose; for instance, to indicate that and antenna is not at the high site, but at the base camp for maintenance.

The table on the left side provides a summary of available antennas broken down by receivers (columns) and current geographical location or antenna type (rows). For instance, twelve antennas in the Inner Array/Central Cluster geographical area have a receiver for B7 operational; and five 7-meter antennas (CM) from the Atacama Compact Array have a receiver for B9 operational. Zero values are painted with a dark, low-contrast color so as to de-emphasize them. This helps users both focus on populated cells, and identify antenna types or geographical areas that lack any observation capabilities, for a given frequency band. For instance, one immediately sees from Figure 1 that B5 and B10 are unavailable no matter the geographical region considered or the antenna type (at the time, neither B5 nor B10 receivers had been commissioned). One can also observe that there is no antenna in any of the three arms (Southern, Western, Pampa La Bola) since at the time, the array was operated with a compact baseline.

\subsection{Detailed Antenna Status}

The Array Elements table is laid out below the Summary section described above. As mentioned earlier, this table is reminiscent of the original spreadsheet crated ad-hoc to track the status of antennas during ALMA's construction phase. It exposes detailed information about each antenna's primary components in tabular form, each row corresponding to one antenna. Information displayed in this table includes the pad on which the antenna is currently positioned, the operation status of primary components as well as that of all receivers, as well as free text comments used to share status information across groups of users and help coordinate tasks.

Rows can be filtered using a conjunction of criteria available from the table header. Figure 2 illustrates this on an example where two filters are cascaded. In addition to filters on status columns, users can also filter columns based on a text search in the different columns showing comments associated with antennas. Complementary to

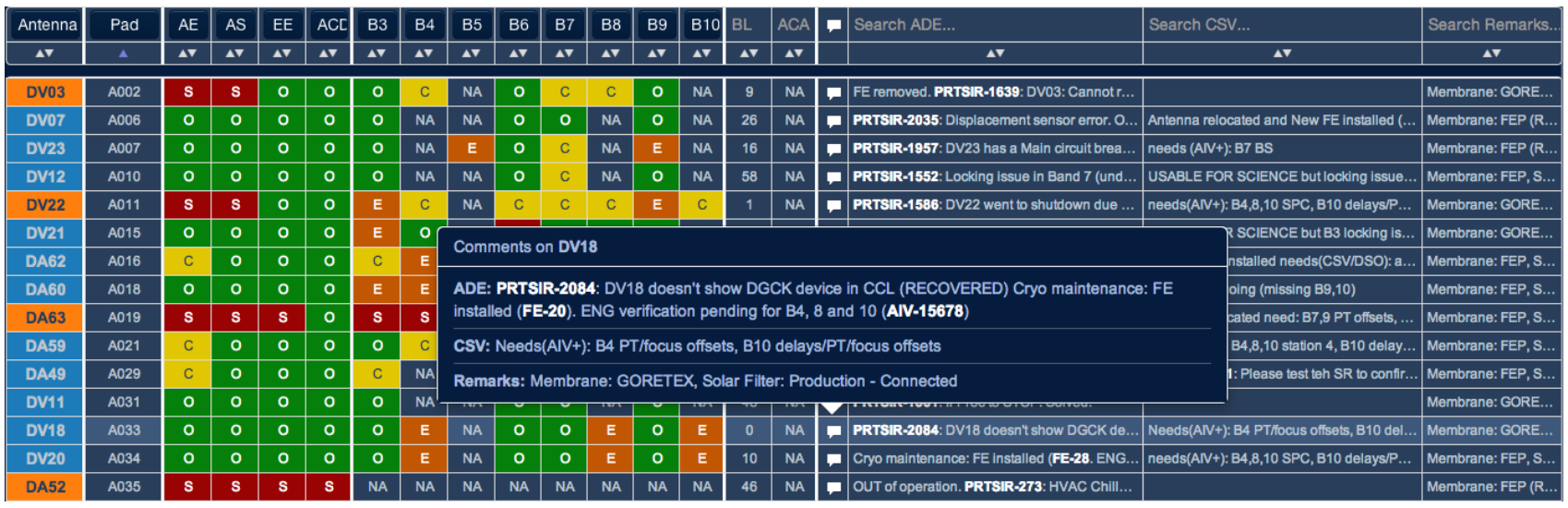

Figure 3. Quickly brushing through comments by moving the cursor in column 


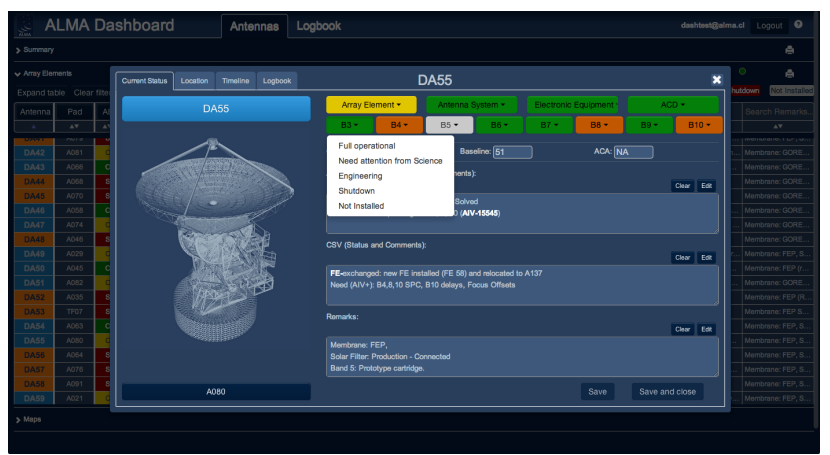

(a)

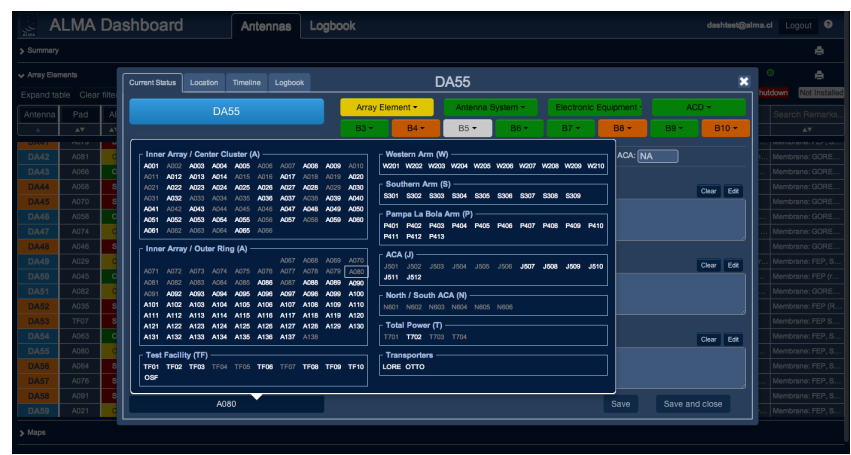

(b)

Figure 4. Detailed view on an antenna. This view lets authenticated users update the status of any variable and edit comments. (a) Changing the status of receiver B3. (b) Assigning the antenna to another pad. Pads already occupied are grayed-out and cannot be selected.

filtering, sorting capabilities are also available and can be applied to any of the columns, letting users reorder antennas according to some specific variable of interest.

Row height in this table is necessarily limited to a single line of text so as to fit as many antennas as possible on a single screen. All 66 antennas will usually not fit, but the goal is to avoid users having to scroll through antennas too much. This means that most of the time, text comments will not fully fit in the corresponding table cells. They are truncated, but users can still efficiently browse through them by brushing with the cursor through a column immediately to the left of the first comments column. As illustrated in Figure 3, the full text of all comments fields for the corresponding antenna are shown simultaneously.

White text labels correspond to tickets in ALMA's issue trackers relevant to a particular antenna. Those ticket identifiers are entered as free text by the Dashboard users. Then the system automatically detects them based on their prefix, e.g., PRTSIR- or AIV-, and displays them as hypertext links so that users can easily get to the corresponding entry in the issue tracker, which is also a Web-based application.

\subsection{Updating Antenna Component Status}

Clicking on a row in the table pops-up a modal window showing additional details about the corresponding antenna. When authenticated, users can update the status of the antenna, as illustrated in Figure 4. All data in this first version of the ALMA Dashboard is input manually by users, because the purpose of the Dashboard is to reflect in a near-real-time manner what subject-matter experts consider to be the current status of an antenna and its components. What this status is depends on human decision making processes involving parameters that might not be captured by any automatic system or monitoring point in the field, and thus requires users to be able to change status variables manually.

\subsection{Geographical View of Antennas}

Finally, users can choose to show a map of antennas below the table (Figure 5). Antennas are overlaid on top of a satellite picture of the AOS site as colored icons reflecting their overall status, giving an overview of antenna availability in the different geographical areas of the array. A similar map is embedded in the Map tab of the antenna modal window. It is centered by default on the corresponding antenna. As all three sections (Summary, ArrayElements, Map) might not always be relevant depending on a given user's tasks and interests, each can be collapsed individually. This saves both screen space and resources when refreshing the Web page.

\subsection{Navigating through Time}

By default, the Dashboard shows the current status of the observatory. But as all edit operations get logged and snapshots taken every hour, users can navigate back in time and inspect the status of the observatory at any arbitrary time in the past. In addition, the antenna modal window features a Timeline tab (Figure 7) that contains a visualization showing the evolution of each status variable over time, as well as when free text comment fields 


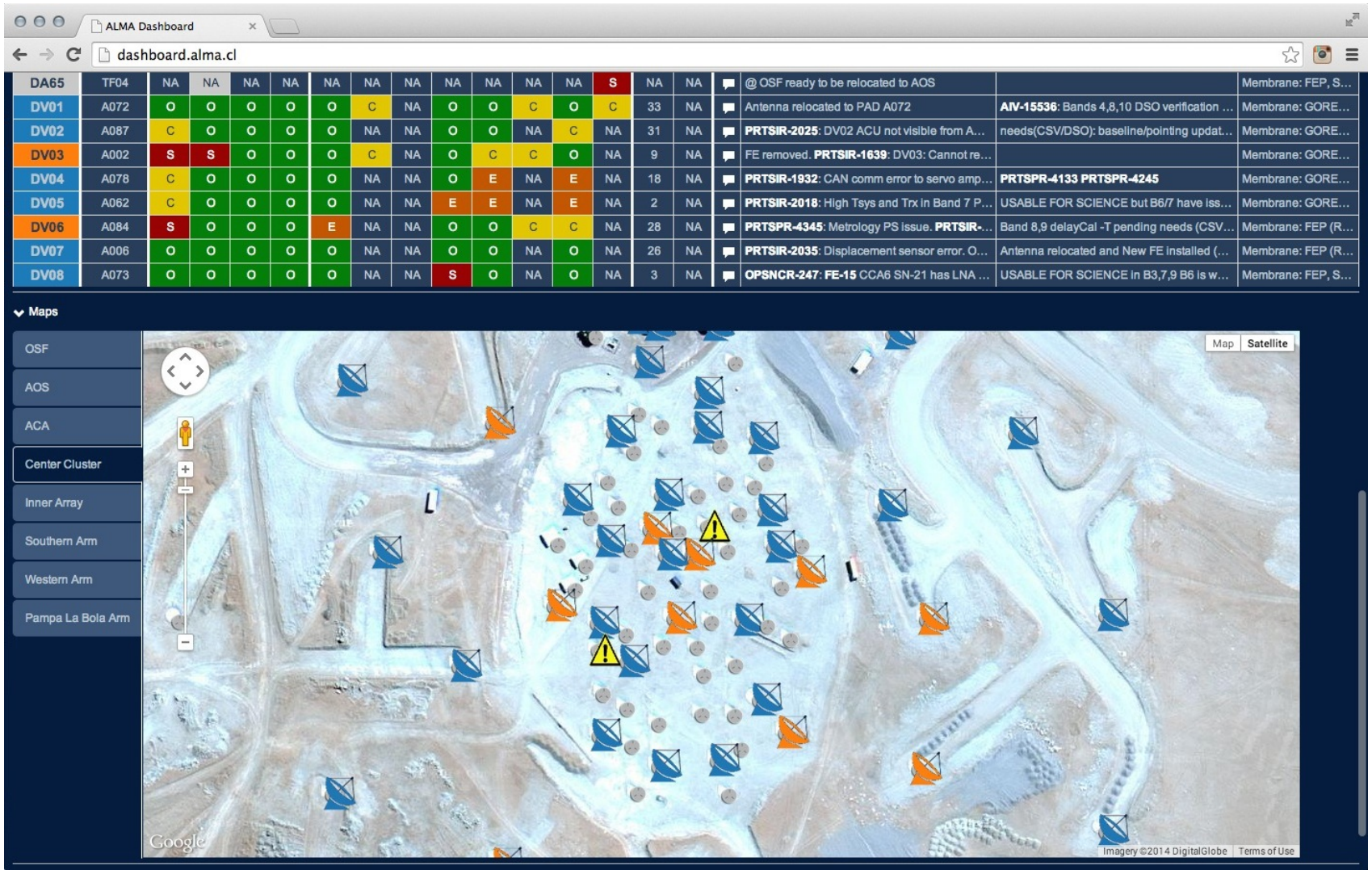

Figure 5. Representation of antennas showing their geographical location using a Google Map widget. Tabs on the left-hand side of the map act as navigation shortcuts to center and zoom the map on a particular area of ALMA.

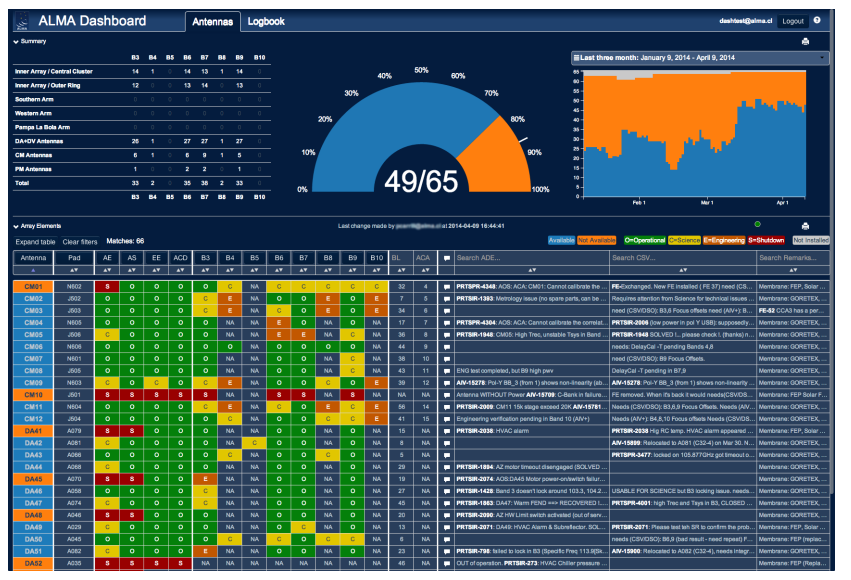

(a)

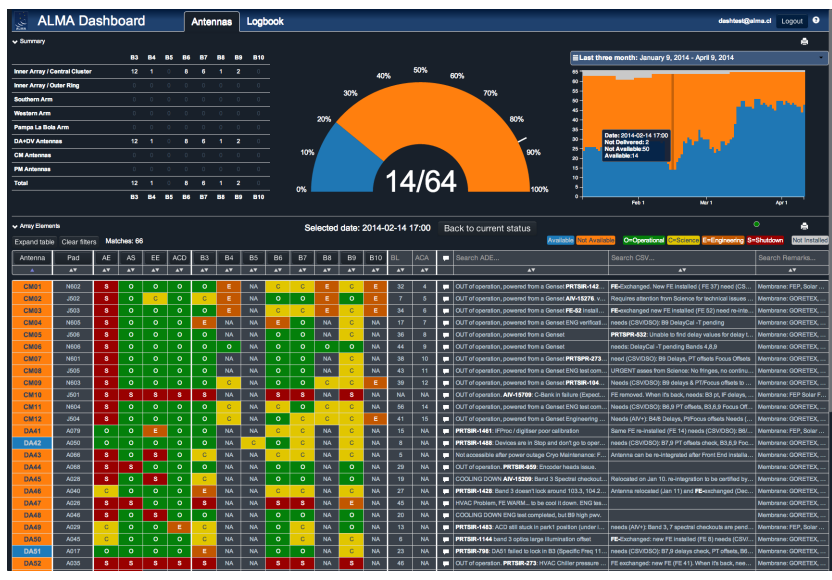

(b)

Figure 6. Navigating back in time: (a) current status, (b) status two months earlier. To avoid users mistaking a past situation for the current one, when navigating back in time, the Dashboard's look and feel is slightly altered: the UI color scheme switches to a mostly grayscale color map except for status variables, and the snapshot's date \& time are prominently displayed right above the antenna table.

were edited. Finally, users can browse through changes made over a given time span by going to the Logbook tab. This can be useful, e.g., when a shift ends and another group of users takes over. By looking at the logbook (Figure 8) for the last 24 hours, a user returning to duty will get a summary of all changes that occurred while she was away. The antenna modal window also features a Logbook tab, that behaves essentially as the general Logbook but only shows events related to the corresponding antenna. 


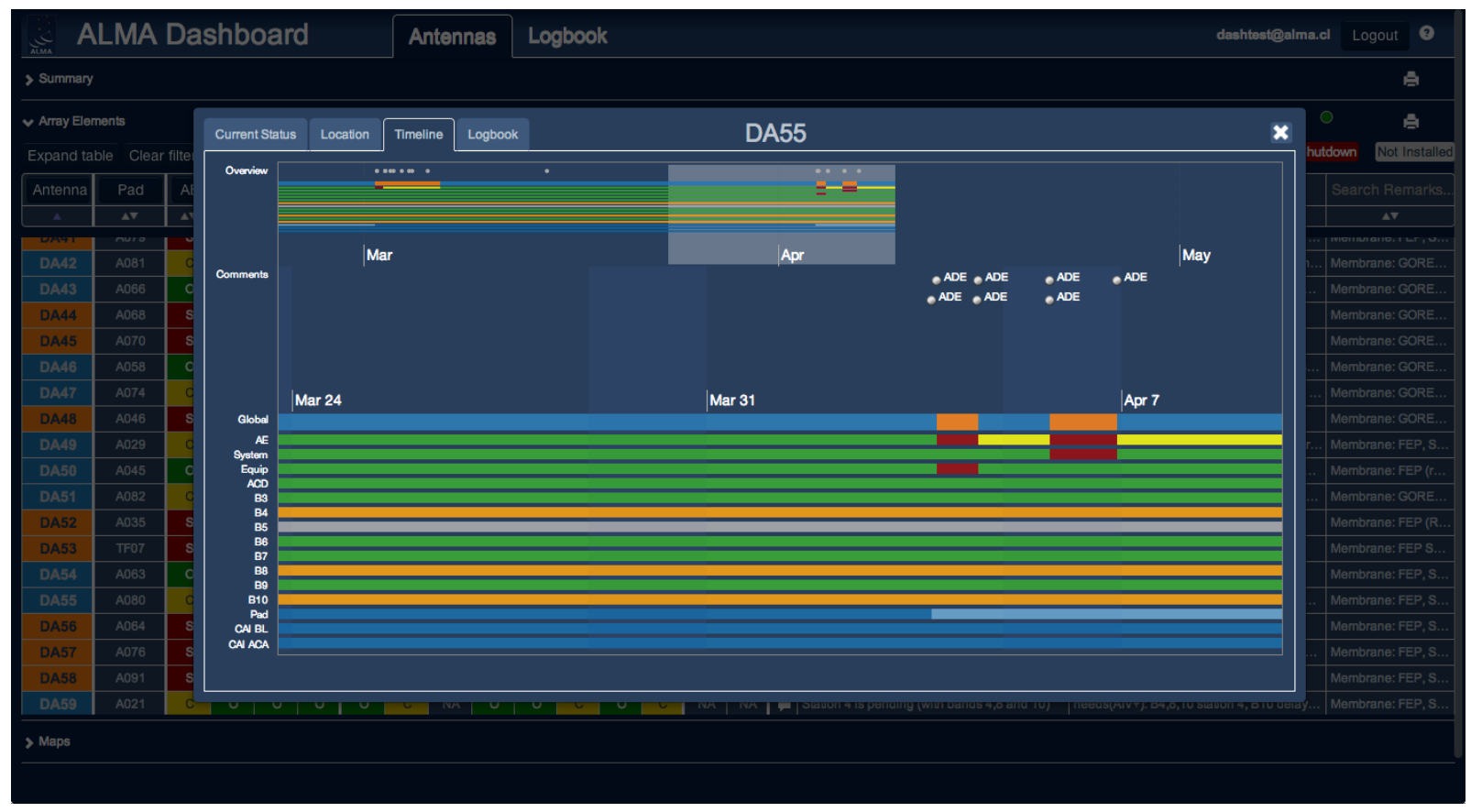

Figure 7. Timeline showing the evolution of each status variable of a given antenna over time. The lower part shows this data in detail. The upper part corresponds to an overview of the same data at a coarser time scale. It can be used to navigate quickly through time. The color palette corresponds to that used in the antenna table on the main page. Punctual events labeled, e.g, ADE, correspond to text editing operations in the corresponding comment field.

\begin{tabular}{|c|c|c|c|c|c|c|c|c|}
\hline$\because A$ & LMA Dashbc & vard & Antennas & Logbook & & dashtertigalma.cl & Logout & (2) \\
\hline Last four: & weeks & & & & & Clear filters & & A \\
\hline AE $\bar{T} \triangle T$ & Time & User & $\Delta \nabla$ & Change Type & Change & & & \\
\hline Fiter & Filter & Fiter & & Filiter & Filter & & & \\
\hline DV06 & $2014-04-0916: 31: 46$ & $=0$ & & $\begin{array}{l}\text { Arrey Element Giobal } \\
\text { Staus }\end{array}$ & Not Available $\rightarrow$ Avallablo & & & \\
\hline DV06 & 201404:09 16:31:45 & $\operatorname{men}=4$ & & ADE Comments & 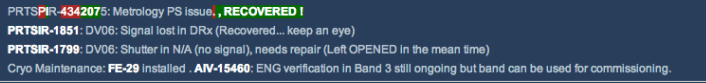 & & & \\
\hline Dvo6 & 2014:04:09 16:31:45 & $=0$ & & Arrey Eement Status & $s \rightarrow 0$ & & & \\
\hline DA49 & 2014-04-09 16:01:35 & $5=0$ & & B3 status & c) $\rightarrow 0$ & & & \\
\hline DA49 & 201404-09 16:01:35 & 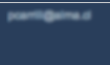 & & ADE Commenis & 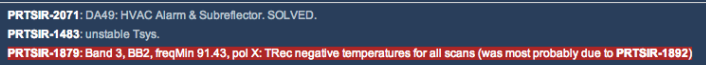 & & & \\
\hline DAA7 & 2014-04:09 15:55:29 & 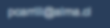 & & ADE Comments & PRTSPIR-43501863: DA47: Warm FEND $\Rightarrow \Rightarrow$ RECOVERED L.... . but need to be re-integrated & & & \\
\hline DA47 & $2014040915: 41: 40$ & 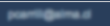 & & ADE Commenis & PRTSPR 4350: DA47: Wam FEND $\Rightarrow$ RECOVERED $1 .$. antenna can., but need to be re-integrated & & & \\
\hline DV10 & 2014-04-09 15:41:25 & $\operatorname{men}=0$ & & ADE Comments & 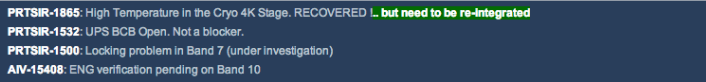 & & & \\
\hline DV18 & 201404:09 15:07:45 & tencosen & & $\begin{array}{l}\text { Amray Element Global } \\
\text { Staus }\end{array}$ & Not Avallable $\rightarrow$ Avallable & & & \\
\hline DV18 & 2014:04:09 15:07:44 & $=0$ & & ADE Commentis & 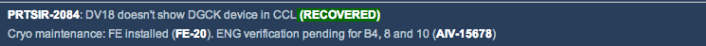 & & & \\
\hline DV18 & 201404:09 15:07:44 & $=0$ & & Sysiem Stetus & $s \rightarrow 0$ & & & \\
\hline DV18 & 2014:04:09 15:07:44 & 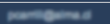 & & Array Element Staus & $\mathbf{s} \rightarrow 0$ & & & \\
\hline DV10 & 2014-04-09 14:51:12 & 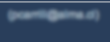 & & $\begin{array}{l}\text { Arrey Eement Giobal } \\
\text { Staus }\end{array}$ & Not Avallable) $\rightarrow$ Avallable & & & \\
\hline DV10 & 201404:09 14:51:11 & $=0$ & & ADE Comments & 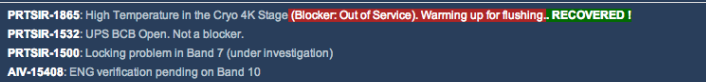 & & & \\
\hline
\end{tabular}

Figure 8. Logbook of events. Users can choose between predefined and custom time spans. Additionally, they can filter for specific types of events, specific antennas, a specific user making changes, or by doing a free text search in the comment fields. The Change columns provides a semi-graphical, colored-diff-like representation of what status variables and text comments changed during each editing operation. 


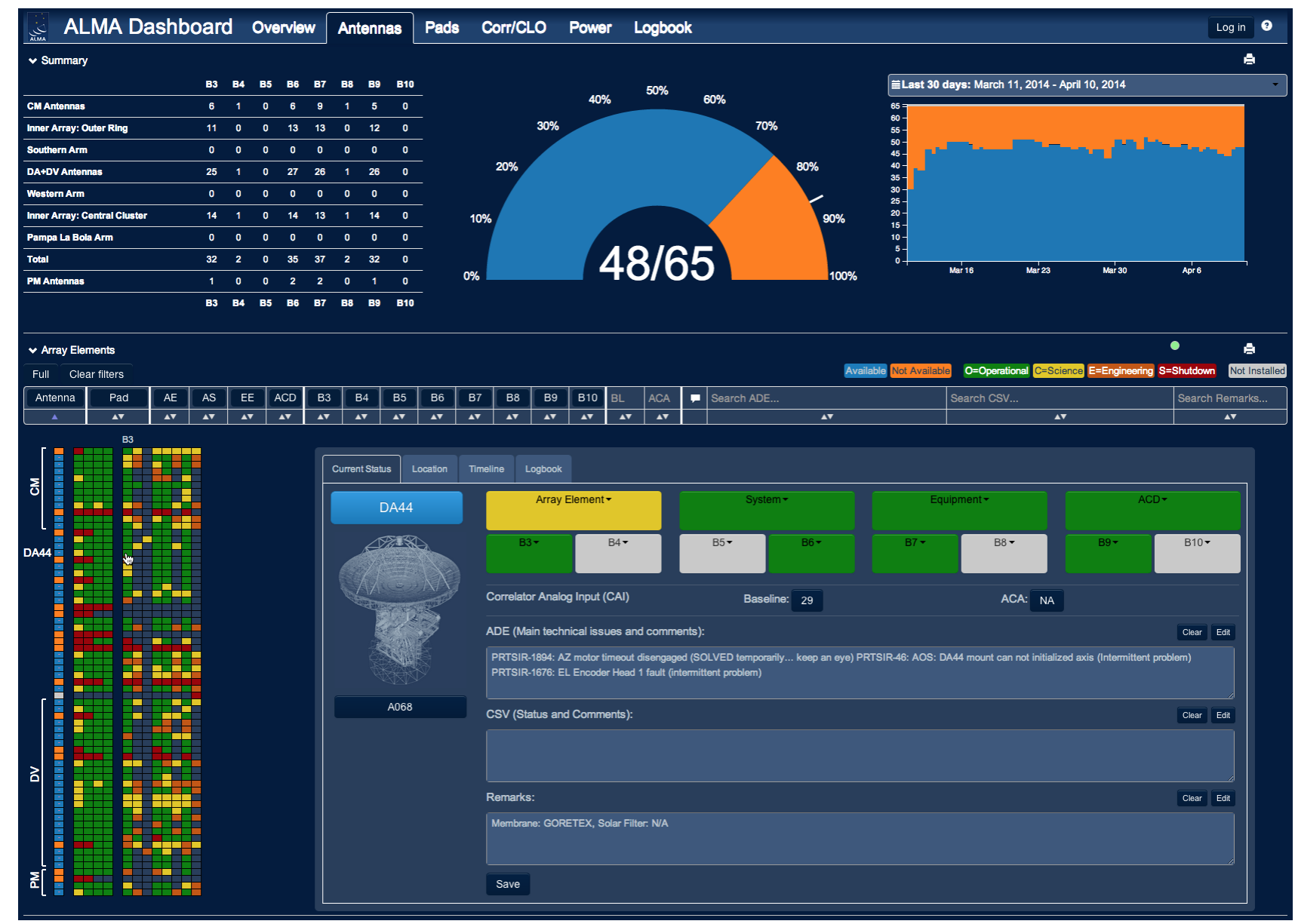

Figure 9. Alternative, more compact view of antennas that fits on a single screen without scrolling while still showing all data about the 66 antennas. The matrix on the left-hand-side enables users to select multiple antennas using the usual spreadsheet-inspired mouse drag and click interaction combined with SHIFT and CONTROL keyboard modifiers to select ranges and non-contiguous rows. Changes can then be applied simultaneously to all selected antennas. As this compact view does not allow to properly label every row and column, the information is shown only when brushing the matrix, and only for the column and row intersected by the mouse cursor. In this example, the cursor is over receiver B3 of antenna DA44. White markers help identify antennas by type (CM, DA, DV, PM), and vertical separators create boundaries between three groups of columns: overall status, main components, and receivers. Selecting an antenna in the matrix updates the detailed antenna panel on the right-hand side, whose content is similar to that of the modal window (Section 3.3) that pops-up when selecting a row in the original antenna table.

\section{EXTENDING THE DASHBOARD}

The first version of the Dashboard was deployed in March 2013 and effectively replaced some previous ad-hoc tools such as the collaborative spreadsheet to track the status of antennas. It went through a series of maintenance releases featuring minor improvements over the following 6 months, taking user feedback into account. As deployment was successful and the user base grew and gained experience with the system, several limitations were identified, as well as opportunities for extension to cover key subsystems beyond antennas. It was thus decided to launch a second design and development phase, following the user-centered design approach described in Section 2. The following sections describe the ongoing work on this second phase of development.

\subsection{A Compact View for Easier Monitoring and Multi-Antenna Editing}

A first limitation identified is that the antenna table on the main page would not fully fit on a single monitor, requiring users to scroll up and down to see all antennas, making interaction somewhat tedious, but more 


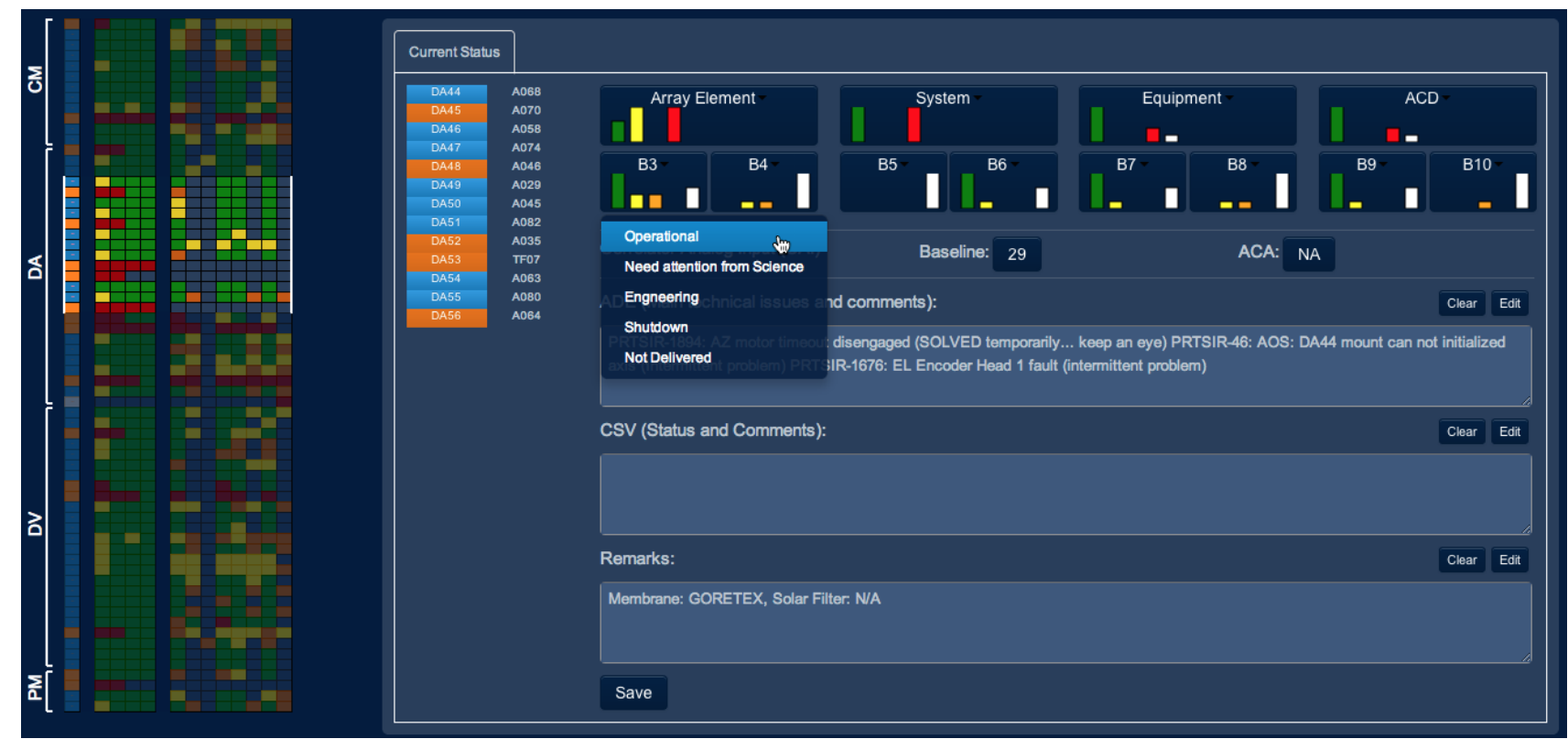

Figure 10. Setting receiver B3 to Operational for antennas DA44 to DA56 with a single edit operation. The small bar charts inside the buttons indicate the distribution of statuses for the corresponding element over the selection of antennas.

importantly preventing the use of the Dashboard for static monitoring. A second limitation reported by endusers was that updating the status of numerous antennas was relatively tedious as they had to be changed one at a time, even if the changes to apply were the same. To address both limitations, we designed an alternative, more compact view of all 66 antennas, that fits on a single screen. Figure 9 illustrates this new view. Beyond enabling static monitoring, this view lets users select multiple antennas and apply changes to all of them simultaneously (Figure 10). This view also features sorting and filtering mechanisms quite similar to those of the original antenna table described in Section 3.2, as illustrated in Figure 11. As opposed to the original antenna table (Section 3.2), filtering rows does not remove them, but simply de-emphasizes them by rendering them with darker, low-contrast colors. We made this choice because we wanted to keep the matrix visually as stable as possible, and because there was no obvious advantage in hiding those rows as this representation is already quite compact. In addition, keeping all rows visible and de-emphasizing those not selected by the filter has the advantage of enabling users to focus on items of interest while still providing some contextual information about the other items. ${ }^{4}$

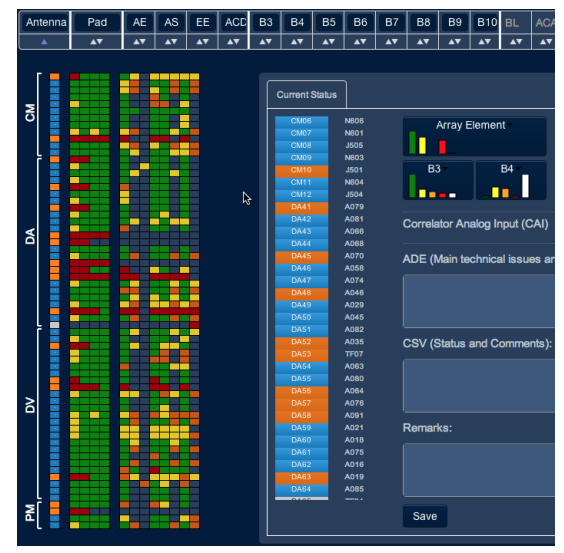

(a)

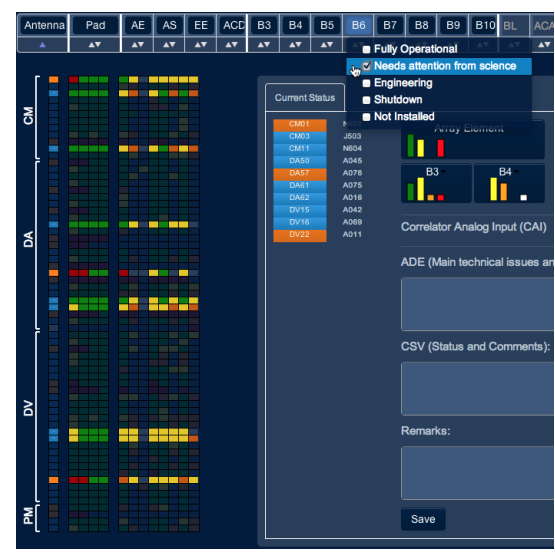

(b)

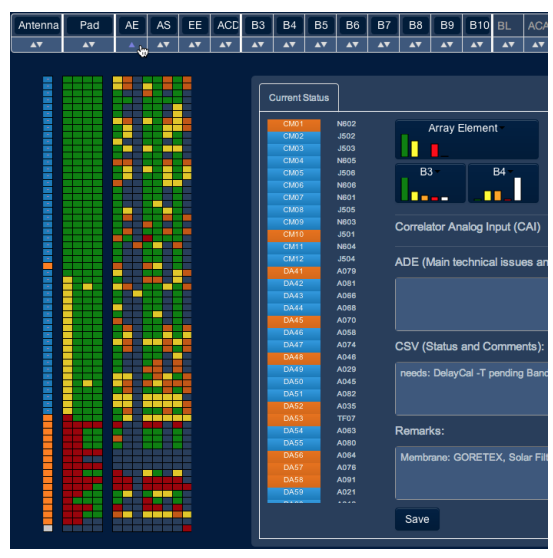

(c)

Figure 11. Filtering and sorting in the compact view: (a) default view, (b) highlighting antennas whose B6 receiver is in status Needs attention from science, (c) sorting rows according to the status of the Antenna Element (AE). 


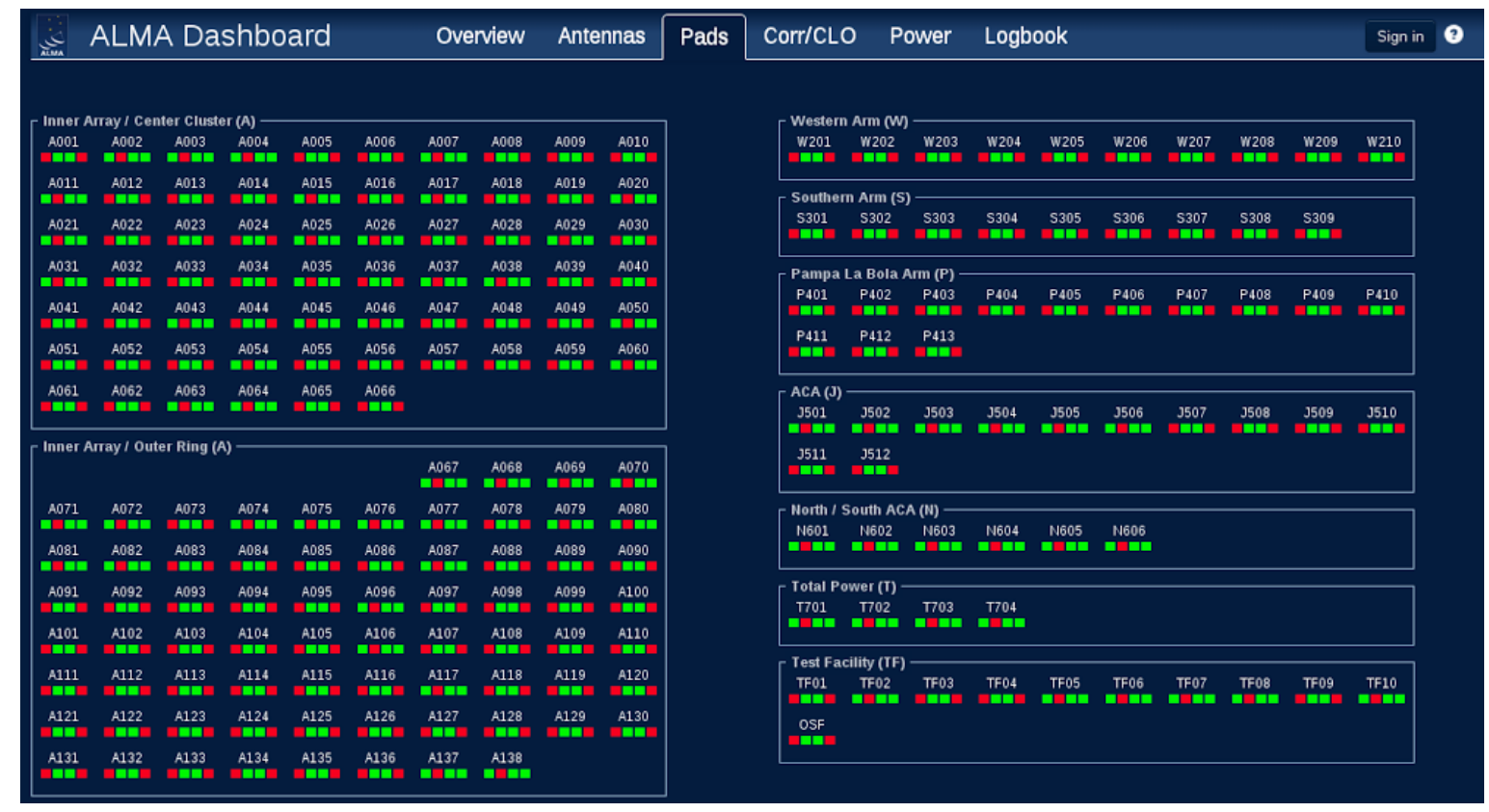

Figure 12. Mockup of the Pads tab. Pads are grouped by geographical area. Four substates are associated with each one of them, and can be toggled by simple clicks on the corresponding colored square: accessibility of pad to ground vehicles, signal vault, power vault, ridges and structure.

\subsection{Further Improving Data Input}

Another way to improve data input, beyond the multi-antenna editing capabilities introduced in the previous section, would be to streamline the process by gathering information from other subsystems. Indeed, some of the information currently being input manually by users in the Dashboard could be fetched from other systems such as specific issue trackers. Feeding data to the Dashboard from these sources is a process that cannot be fully automated, because even if those sources hold structured data about issues related to the status of antennas and other elements of the observatory, some ambiguities can remain. Most importantly, the process requires human supervision because it might not always be relevant to update a component's status based on changes made in an issue tracker.

Our goal is thus not to feed data directly to the Dashboard's database, but to feed a queue of changes that first have to be validated. Suggestions for modifications to Dashboard status data would be generated from this queue and presented to workers in charge of the Dashboard. Based on their knowledge of the system and external information they may have, they can decide either to apply the suggested changes automatically simply by validating them, to modify them before they get applied, or to ignore them. The first and third option would only require clicking on a button, which should make the task of reviewing the queue of suggested updates a fairly smooth and efficient process.

Implementing such a solution requires making changes both to the Dashboard and to the source systems from which data would be fetched to generate the suggestions. Two strategies are currently being considered: 1. implementing a push mechanism, where each of these source systems makes, e.g., an HTTP request to the Dashboard using its REST API whenever transitions in their workflows or any kind of change associated with a potential antenna status update happens; or 2. implementing a pull mechanism, where the Dashboard regularly polls these systems for any new tracker issue creation or update that might generate a suggestion for antenna status update. The technical feasibility of these solutions is currently being evaluated.

\subsection{Tracking Additional Equipment}

So far the Dashboard has focused only on what is considered to be the main components of the observatory: the antennas. But other systems also play important roles: the correlator, the central local oscillator, the power 


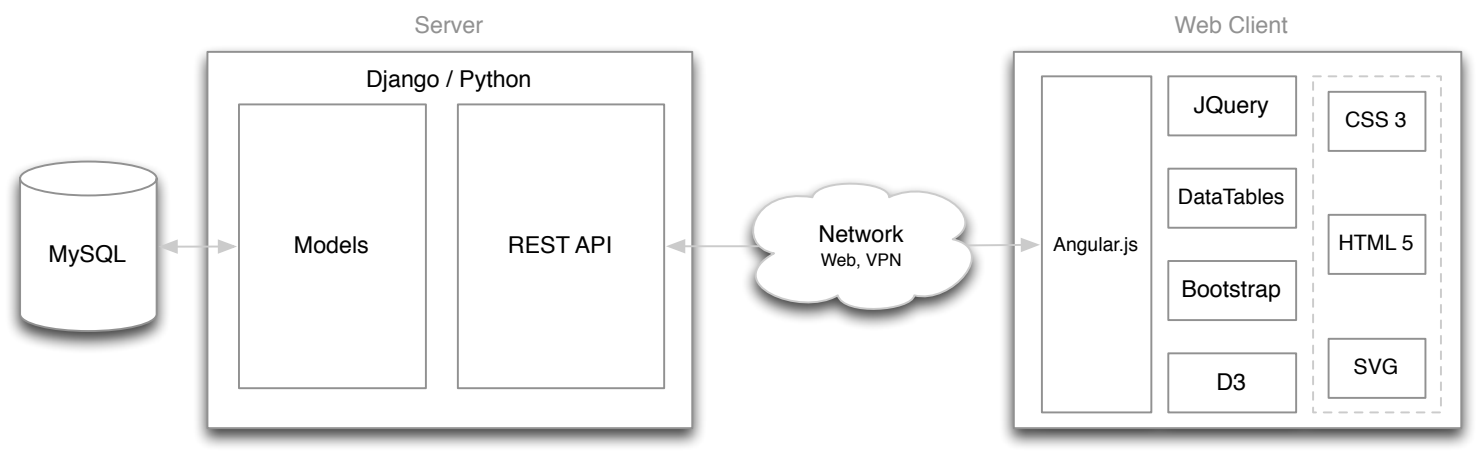

Figure 13. Dashboard architecture.

distribution network, the pads holding the antennas. All of these are complex systems with multiple sub-states that ALMA personnel need to keep track of during operations and maintenance.

Following an approach similar to that used for antennas, new tabs are being added to the Dashboard for these systems, as shown at the top of Figure 9. These are all work-in-progress. Figure 12 illustrates the current mockup for the Pads tab, where four sub-states are associated with each pad.

In addition to these supplemental systems, the new version of the ALMA Dashboard will also feature an overview page, summarizing the status of all main systems in one diagram, as well as a widget to display and post important, site-wide messages including incidents, unusual weather conditions or reminders about maintenance actions that affect multiple groups.

\section{IMPLEMENTATION}

As illustrated in Figure 13, the Dashboard is implemented as a typical client-server Web application. Multiple clients can connect to the server and make concurrent modifications. Users need to be authenticated to update the data. The data are stored in a MySQL database as snapshots taken every hour, complemented with a log of all update events performed by users. On the server side, Django is used as the Web framework connecting to the database. It exposes the data to clients through a REST API. On the client side, the Dashboard makes heavy use of Javascript libraries, including JQuery, DataTables, and D3 ${ }^{5}$ for the interactive data visualization components. The UI eventually gets rendered as a combination of HTML 5 content, SVG graphics and CSS 3 styling instructions.

\section{REUSE OF DASHBOARD DATA IN OTHER SYSTEMS}

As the Dashboard exposes its data to clients using a REST API, third-party clients, not necessarily running in a Web browser, can query it and make use of its data (read-only) for arbitrary purposes. It is the case, for instance, of the latest version of the ZVTM-based ${ }^{6}$ Antenna Navigator $^{1}$ that provides a multi-scale ${ }^{7}$ interactive map of the AOS for the Operations Monitoring and Control (OMC) software that runs in the observatory's control room. This component now enables operators to identify on the AOS map antennas that meet particular criteria based on data stored in the Dashboard, such as, e.g., all antennas available for science having B3 and B6 receivers operational. This should eventually help operators create arrays of antennas. Indeed, they formerly had to look into different places to find the relevant information: the collaborative spreadsheet, real-time monitoring data in the OMC to check the online status of antennas; once this information collected, they would then create arrays using a dedicated user interface component. With the new Antenna Navigator, that was already showing online status information based on the real-time monitoring data, and that is now able to fetch Dashboard data, operators will be able to perform all operations from a single interface component that makes all this information readily available.

Figure 14 shows an interactive multi-scale visualization that was derived from the Antenna Navigator and will be used for array configuration planning. It provides users with a dynamic query interface to inspect different antenna configurations, highlighting particular geographical regions (pads) and antennas meeting specific criteria. 


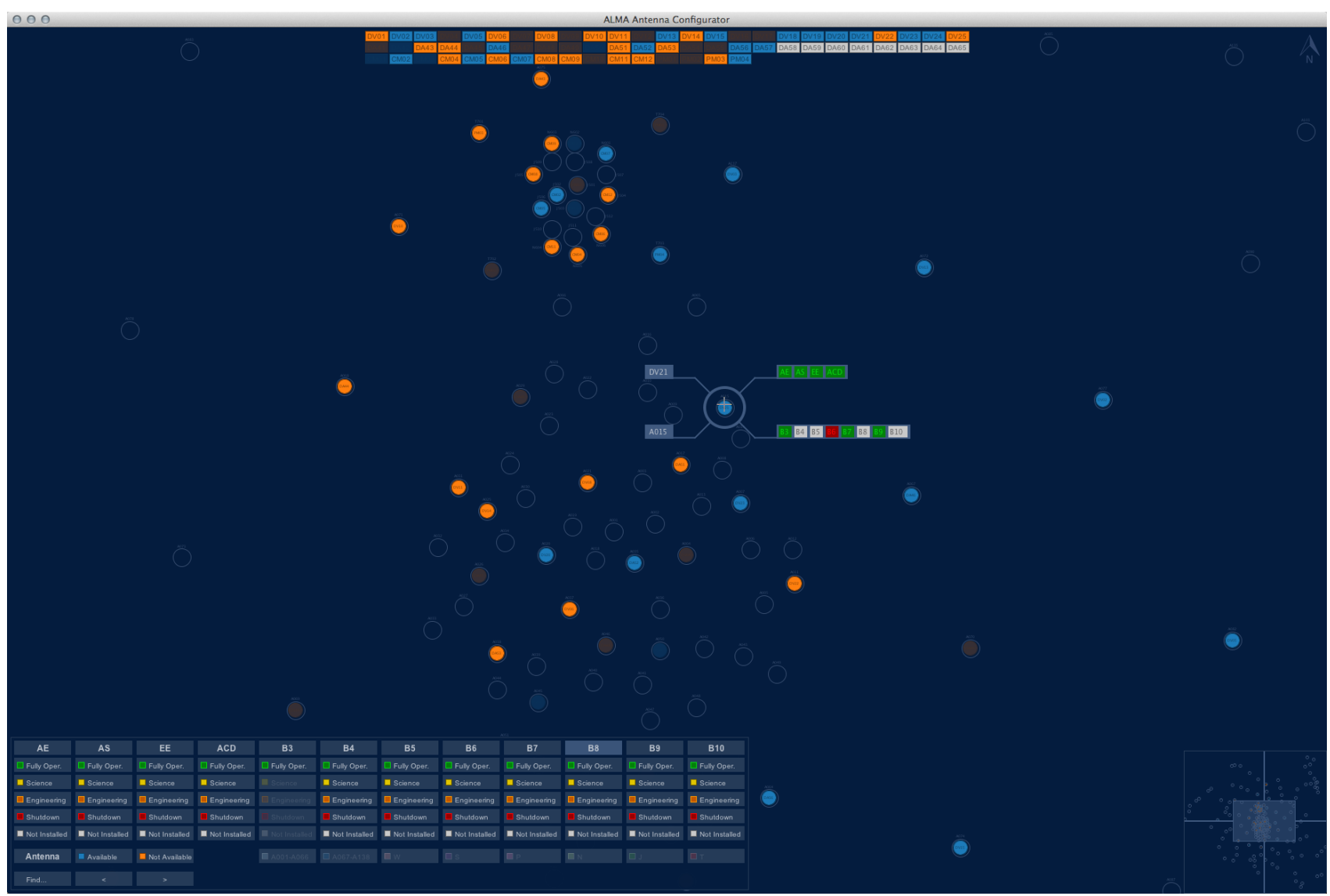

Figure 14. Java-based interactive visualization tool for array configuration, making use of Dashboard data through the REST API. The view is configured to emphasize antennas that have a B3 receiver operational.

\section{ACKNOWLEDGMENTS}

We wish to thank all astronomers, engineers and operators who participated in discussions and provided us with insightful comments and ideas, including: Hector Alarcon, Denis Barkats, Emilio Barrios, Pablo Carillo, Maurizio Chavan, Vasco Cortez, Antonio Hales, Daniel Kent, Ruediger Kneissl, Stéphane Leon Tanne, Gianni Marconi, Rodrigo Olguin, Joaquin Penroz, Diego Rojas, Norman Saez, Masao Saito, Marcus Schilling, Nick Whyborn.

\section{REFERENCES}

[1] Pietriga, E., Cubaud, P., Schwarz, J., Primet, R., Schilling, M., Barkats, D., Barrios, E., and Vilaro, B. V., "Interaction Design Challenges and Solutions for ALMA Operations Monitoring and Control," in [Proceedings of the Astronomical Telescopes and Instrumentation conference: Software and Cyberinfrastructure for Astronomy II], SPIE (2012).

[2] Schwarz, J., Pietriga, E., Schilling, M., and Grosbøl, P., "Goodbye to WIMPs: A Scalable Interface for ALMA Operations," in [ADASS '10: Proceedings of the Astronomical Data Analysis Software and Systems], ASP (2010).

[3] Schilling, M., Primet, R., Pietriga, E., and Schwarz, J., "Human Computer Interaction in the ALMA Control Room," in [ADASS '11: Proceedings of the Astronomical Data Analysis Software and Systems], 137-140, ASP (2011).

[4] Furnas, G. W., "A Fisheye Follow-up: Further Reflections on Focus + Context," in [Proceedings of the SIGCHI Conference on Human Factors in Computing Systems], CHI '06, 999-1008, ACM (2006).

[5] Bostock, M., Ogievetsky, V., and Heer, J., "D3 Data-Driven Documents," IEEE Transactions on Visualization and Computer Graphics 17, 2301-2309 (Dec. 2011).

[6] Pietriga, E., "A Toolkit for Addressing HCI Issues in Visual Language Environments," in [IEEE Symposium on Visual Languages and Human-Centric Computing (VL/HCC'05)], 145-152, IEEE Computer Society (2005).

[7] Cockburn, A., Karlson, A., and Bederson, B. B., "A Review of Overview + Detail, Zooming, and Focus + Context Interfaces," ACM Comput. Surv. 41, 2:1-2:31 (Jan. 2009). 